Honam Mathematical J. 35 (2013), No. 2, pp. 211-223

http://dx.doi.org/10.5831/HMJ.2013.35.2.211

\title{
MINIMUM PERMANENTS ON DOUBLY STOCHASTIC MATRICES WITH PRESCRIBED ZEROS
}

\author{
SEOK-Zun SONG
}

\begin{abstract}
We consider permanent function on the faces of the polytope of certain doubly stochastic matrices, whose nonzero entries coincide with those of fully indecomposable square $(0,1)$-matrices containing identity submatrix. We determine the minimum permanents and minimizing matrices on the given faces of the polytope using the contraction method.
\end{abstract}

\section{Introduction and preliminaries}

Let $\Omega_{n}$ be the polyhedron of $n \times n$ doubly stochastic matrices, that is, the $n \times n$ nonnegative matrices whose row and column sums are all equal to 1 . The permanent of an $n \times n$ matrix $A=\left[a_{i j}\right]$ is defined by

$$
\operatorname{per}(A)=\sum_{\sigma} a_{1 \sigma(1)} \cdots a_{n \sigma(n)}
$$

where $\sigma$ runs over all permutations of $\{1,2, \ldots, n\}$.

Let $J_{r, s}$ denote the $r \times s$ matrix all of whose entries are 1 . In 1981 Egorycev [3] and Falikman [4] proved the van der Waerden permanent conjecture: If $A \in \Omega_{n}$, then

$$
\operatorname{per}(A) \geq \operatorname{per}\left(\frac{1}{n} J_{n, n}\right) .
$$

The techniques of Egorycev have been used, with some success, for determination of minimum permanents in various faces of $\Omega_{n}$ (See [2], [5] -[9]). The key technique is replacing rows (or columns) of a matrix with

Received March 25, 2013. Accepted April 6, 2013.

2010 Mathematics Subject Classification. Primary 15A15, 15A51.

Key words and phrases. permanent, doubly stochastic matrix, barycentric, cohesive matrix.

This research was supported by Basic Science Research Program through the National Research Foundation of Korea (NRF) funded by the Ministry of Education, Science and Technology (No. 2010-0003011). 
minimum permanent by their average without altering its permanent. Unfortunately, the presence of fixed zeros restricts the use of this technique. Indeed this tool is not available at all in the case of faces which consist of matrices with at least one fixed zero in each row and column. In this paper we use this technique in some parts of proofs.

Let $D=\left[d_{i j}\right]$ be an $n$-square nonnegative matrix, and let

$$
\Omega(D)=\left\{X=\left[x_{i j}\right] \in \Omega_{n} \mid x_{i j}=0 \text { whenever } d_{i j}=0\right\} .
$$

Then $\Omega(D)$ is a face of $\Omega_{n}$, and since it is compact, $\Omega(D)$ contains a minimizing matrix $A$ such that $\operatorname{per}(A) \leq \operatorname{per}(X)$ for all $X \in \Omega(D)$.

Brualdi [1] defined an $n$-square $(0,1)$ matrix $D$ to be cohesive if there is a matrix $Z$ in the interior of $\Omega(D)$ for which

$$
\operatorname{per}(Z)=\min \{\operatorname{per}(X) \mid X \in \Omega(D)\} .
$$

And he defined an $n$-square $(0,1)$ matrix $D$ to be barycentric if

$$
\operatorname{per}(b(D))=\min \{\operatorname{per}(X) \mid X \in \Omega(D)\},
$$

where the barycenter $b(D)$ of $\Omega(D)$ is given by

$$
b(D)=\frac{1}{\operatorname{per}(D)} \sum_{P \leq D} P
$$

where the summation extends over the set of all permutation matrices $P$ with $P \leq D$ and $\operatorname{per}(D)$ is their number.

Let $I_{n}$ denote the identity matrix of order $n$ and $0_{k}$ the $k \times k$ zero matrix.

In [9], Song considered a matrix $V_{m, n}=\left[\begin{array}{cc}J_{m, m} & J_{m, n} \\ J_{n, m} & I_{n}\end{array}\right]$ and suggested determining the minimum permanents and minimizing matrices on $\Omega\left(V_{m, n}\right)$ for $m \geq 2$, and $n \geq 3$. This face $\Omega\left(V_{m, n}\right)$ is extended one of $\Omega\left(W_{n}\right)$ in Theorem 5 in [1].

Brualdi ([1]) determined the minimum permanent and minimizing matrix on $\Omega\left(V_{1, n-1}\right)$. Song determined the minimum permanents on $\Omega\left(V_{3, n}\right)$ in [8] and on $\Omega\left(V_{m, 3}\right)$ in [7], respectively. In [6] the authors determined the minimum permanents for various cases and gave a conjecture. 
In this paper, we consider the faces $\Omega\left(V_{4, n}\right)$ and $\Omega\left(U_{4, n}\right)$, where $U_{4, n}=$ $\left[\begin{array}{cc}0_{4,4} & J_{4, n} \\ J_{n, 4} & I_{n}\end{array}\right]$. We show that $U_{4, n}$ is both cohesive and barycentric and determine the minimum permanents and minimizing matrices on the face $\Omega\left(V_{4, n}\right)$ of $\Omega_{4+n}$ for $n \geq 6$ using the contraction method, which was obtained under certain assumption in [6]. We show that the Conjecture in [6] holds in some part. Our results contribute toward characterizations of cohesive and barycentric matrices respectively, which were asked for in $[1]$.

Recall that an $n$-square nonnegative matrix is said to be fully indecomposable if it contains no $k \times(n-k)$ zero submatrix for $k=1, \cdots, n-1$.

For a matrix $A$, let $A(i, j, \ldots, k \mid l, m, \ldots, n)$ denote the submatrix obtained from $A$ by deleting rows $i, j, \ldots, k$, and columns $l, m, \ldots, n$. In particular, we simplify the notation $A(i, j, \ldots, k \mid i, j, \ldots, k)$ to $A(i, j, \ldots, k)$.

We use the following well-known Lemma ([5]).

Lemma 1.1. ([5]) Let $D=\left[d_{i j}\right]$ be an $n$-square fully indecomposable $(0,1)$ matrix, and $A=\left[a_{i j}\right]$ be a minimizing matrix on $\Omega(D)$. Then $A$ is fully indecomposable, and for $(i, j)$ such that $d_{i j}=1$,

$$
\begin{aligned}
& \operatorname{per}(A(i \mid j))=\operatorname{per}(A) \quad \text { if } a_{i j}>0, \\
& \operatorname{per}(A(i \mid j)) \geq \operatorname{per}(A) \quad \text { if } a_{i j}=0 .
\end{aligned}
$$

\section{The Cohesiveness of $U_{4, n}$}

In this section, we consider the $(4+n)$-square $(0,1)$ matrix $U_{4, n}$ and we show that this matrix is cohesive for $n \geq 6$.

If a column $j$ of an $n \times n$ matrix $A$ contains exactly $k$ nonzero entries $(2 \leq k \leq n)$, say in rows $r_{1}, \ldots, r_{k}$, then the $(n-1)$ square matrix $G C(A)$ obtained from $A$ by replacing rows $r_{2}, \ldots, r_{k}$ with $\frac{1}{k-1}\left(r_{1}+r_{2}+\ldots+r_{k}\right)$ and deleting row $r_{1}$ and column $j$ is called a generalized contraction of $A$.

Lemma 2.1. ([8]) Suppose $D \in \Omega_{n}$ is fully indecomposable and has a column (row) with exactly $k$ positive entries and those $k$ rows (columns) have the same zero pattern. Let $A$ be a minimizing matrix on $\Omega(D)$. 
Then $G C(A)$ is fully indecomposable, and there is a minimizing matrix $\overline{G C(A)} \in \Omega(G C(A))$ satisfying

$$
\operatorname{per}(A)=\left(\frac{k-1}{k}\right)^{k-1} \operatorname{per}(G C(A)) \geq\left(\frac{k-1}{k}\right)^{k-1} \operatorname{per}(\overline{G C(A)}) .
$$

Lemma 2.2. ([8]) For $n \geq 5, U_{3, n}=\left[\begin{array}{cc}0_{3} & J_{3, n} \\ J_{n, 3} & I_{n}\end{array}\right]$ is barycentric and the minimum permanent on $\Omega\left(U_{3, n}\right)$ is $\frac{6(n-1)(n-2)(n-3)^{n-3}}{n^{n+2}}$.

Theorem 2.3. For $n \geq 5$, we show that $U_{4, n}$ is a cohesive matrix.

Proof. Let $B_{4, n}$ be a minimizing matrix on $\Omega\left(U_{4, n}\right)$. Since the first 4 columns and first 4 rows of $U_{4, n}$ are the same, we can use the averaging method(see [3] or [6]) on those columns and rows of $B_{4, n}$, respectively. Thus we can write $B_{4, n}$ as follows:

$$
B_{4, n}=\left[\begin{array}{c|cccc}
0_{4} & \overline{\mathbf{b}}_{1} & \overline{\mathbf{b}}_{2} & \ldots & \overline{\mathbf{b}}_{n} \\
\hline \overline{\mathbf{b}}_{1}^{t} & x_{1} & & & 0 \\
\overline{\mathbf{b}}_{2}^{t} & & x_{2} & & \\
\vdots & 0 & & \ddots & \\
\overline{\mathbf{b}}_{n}^{t} & & & & x_{n}
\end{array}\right],
$$

where $\overline{\mathbf{b}}_{i}\left(\overline{\mathbf{b}}_{i}^{t}\right)$ is a column (row) vector with $b_{i}$ as all its entries for $i=$ $1,2, \cdots, n$.

Since the permanent value is invariant under the interchange of rows (and columns, respectively), we may assume that

$$
\left.b_{i+1} \leq b_{i} \quad \text { (i.e. } \quad x_{i+1} \geq x_{i}\right)
$$

for $i=1,2, \cdots, n-1$, without loss of generality. Since $U_{3, n}$ is fully indecomposable, each

$$
b_{i} \neq 0
$$

for $i=1, \cdots, n$.

Suppose $x_{1}=0$. Then the fourth row and column of $B_{4, n}$ have exactly 4 nonzero entries. Thus we can obtain a generalized contraction $G C\left(B_{4, n}\right)$ of $B_{4, n}$. Since the third row of $G C\left(B_{4, n}\right)$ has exactly 3 nonzero 
entries, we can obtain its generalized contraction $G C\left(G C\left(B_{4, n}\right)\right)$, which is contained in $\Omega\left(U_{3, n-1}\right)$. Using Lemmas 2.1 and 2.2 , we have

$$
\begin{aligned}
\operatorname{per}\left(B_{4, n}\right) & =\left(\frac{3}{4}\right)^{3} \operatorname{per}\left(G C\left(B_{4, n}\right)\right) \\
& \geq\left(\frac{3}{4}\right)^{3}\left\{\left(\frac{3}{4}\right)^{3} b\left(U_{3, n-1}\right)\right\} \\
& =\left(\frac{3}{4}\right)^{6} \cdot \frac{6(n-2)(n-3)(n-4)^{n-4}}{(n-1)^{n+1}} .
\end{aligned}
$$

But $\operatorname{per}\left(B_{4, n}\right)$ is less than or equal to the permanent of the barycenter $b\left(U_{4, n}\right)$. That is,

$$
\begin{aligned}
& \operatorname{per}\left(B_{4, n}\right) \leq \operatorname{per}\left(b\left(U_{4, n}\right)\right) \\
& =4 \cdot \frac{1}{n}\left[(n-1) \cdot \frac{1}{n} \cdot \frac{3}{n} \cdot(n-2) \cdot \frac{1}{n} \cdot \frac{2}{n} \cdot(n-3) \cdot \frac{1}{n} \cdot \frac{1}{n}\right]\left(\frac{n-4}{n}\right)^{n-4} \\
& =\frac{4 !(n-1)(n-2)(n-3)(n-4)^{n-4}}{n^{n+3}}
\end{aligned}
$$

If we divide the last value in (2.5) by the last value in (2.4), then we can show that the result is less than 1 by a direct calculation using Mathematica Program for $n \geq 5$. Thus we have a contradiction from the inequalities in (2.4) and (2.5). Hence $x_{1}$ is not zero. By (2.2), each

$$
x_{i} \neq 0
$$

for $i=1, \cdots, n$. Hence $U_{4, n}$ is cohesive by (2.3) and (2.6).

Remark 2.4. In [6], the cohesiveness of $U_{m, n}$ was not proved. And the fact that $x_{i}>0$ was assumed in the proof of the Theorem 2.1 in [6]. But this fact that each $x_{i}>0$ is essential for the determining of the minimum permanents on $\Omega\left(U_{4, n}\right)$.

\section{Minimum permanents on $\Omega\left(U_{4, n}\right)$}

In this section, we show that $U_{4, n}$ is a barycenteric matrix and hence we determine the minimum permanents on $\Omega\left(U_{4, n}\right)$ for $n \geq 5$ using the results in Theorem 2.3.

Theorem 3.1. For $n \geq 5$, the minimum permanent on $\Omega\left(U_{4, n}\right)$ is

$$
\frac{4 !(n-1)(n-2)(n-3)(n-4)^{n-4}}{n^{n+3}} .
$$

which occurs at the barycenter $b\left(U_{4, n}\right)$. 
Proof. Let $B_{4, n}$ be a minimizing matrix on $\Omega\left(U_{4, n}\right)$. Then $B_{4, n}$ has the form of $B_{4, n}$ in (2.1) as the proof of Theorem 2.3. Without loss of generality, we also assume

$$
b_{i+1} \leq b_{i}\left(\text { i.e. } x_{i+1} \geq x_{i}\right)
$$

for $i=1,2, \cdots, n-1$. Since $U_{4, n}$ is a cohesive matrix by Theorem 2.3, each $b_{i}$ and $x_{i}$ are positive for $i=1,2, \cdots, n$.

Now, we will show that all $b_{i}$ are the same. Suppose to the contrary that $b_{1}>b_{n}$. Then we have $\operatorname{per} B_{4, n}=\operatorname{per} B_{4, n}(1 \mid 5)=\operatorname{per} B_{4, n}(1 \mid$ $n+4)$, from Lemma 1.1 since all $b_{i}>0$. But

$$
\operatorname{per} B_{4, n}(1 \mid 5)=\left(4 b_{1}\right)\left\{\left(3 b_{n}\right)^{2} \operatorname{per} B_{4, n}(1,2,5, n)+x_{n} \operatorname{per} B_{4, n}(1,5, n)\right\} \text {, }
$$

and

$$
\operatorname{per} B_{4, n}(1 \mid n)=\left(4 b_{n}\right)\left\{\left(3 b_{1}\right)^{2} \operatorname{per} B_{4, n}(1,2,5, n)+x_{1} \operatorname{per} B_{4, n}(1,5, n)\right\} .
$$

From these values, using $x_{i}=1-4 b_{i}$ for $i=1$ and $n$, we have

$$
9 b_{1} b_{n} \operatorname{per} B_{4, n}(1,2,5, n)=\operatorname{per} B_{4, n}(1,5, n) .
$$

Since all $x_{i}>0$, we have

$$
\operatorname{per} B_{4, n}=\operatorname{per} B_{4, n}(5 \mid 5)=\operatorname{per} B_{4, n}(n \mid n),
$$

from Lemma 1.1.

But

$$
\operatorname{per} B_{4, n}(5 \mid 5)=\left(4 b_{n}\right)^{2} \operatorname{per} B_{4, n}(1,5, n)+x_{n} \operatorname{per} B_{4, n}(5, n),
$$

and

$$
\operatorname{per} B_{4, n}(n \mid n)=\left(4 b_{1}\right)^{2} \operatorname{per} B_{4, n}(1,5, n)+x_{1} \operatorname{per} B_{4, n}(5, n) \text {. }
$$

From these values, using $x_{i}=1-4 b_{i}$ for $i=1$ and $n$, we have

$$
4\left(b_{1}+b_{n}\right) \operatorname{per} B_{4, n}(1,5, n)=\operatorname{per} B_{4, n}(5, n) .
$$

Let us calculate the $\operatorname{per} B_{4, n}$ by expanding along the n-th and 5 -th columns. Then we have

$$
\begin{aligned}
\operatorname{per} & B_{4, n} \\
= & \left(4 b_{n}\right)^{2} \operatorname{per} B_{4, n}(1, n)+x_{n} \operatorname{per} B_{4, n}(n \mid n) \\
= & \left(4 b_{n}\right)^{2}\left\{\left(3 b_{1}\right)^{2} \operatorname{per} B_{4, n}(1,2,5, n)+x_{1} \operatorname{per} B_{4, n}(1,5, n)\right\} \\
& +x_{n}\left\{\left(4 b_{1}\right)^{2} \operatorname{per} B_{4, n}(1,5, n)+x_{1} \operatorname{per} B_{4, n}(5, n)\right\} \\
= & 4^{2} \cdot 3^{2} \cdot b_{1}^{2} b_{n}^{2} \operatorname{per} B_{4, n}(1,2,5, n)+16 b_{n}^{2}\left(1-4 b_{1}\right) \operatorname{per} B_{4, n}(1,5, n) \\
& +16 b_{1}^{2}\left(1-4 b_{n}\right) \operatorname{per} B_{4, n}(1,5, n)+\left(1-4 b_{n}\right)\left(1-4 b_{1}\right) \operatorname{per} B_{4, n}(5, n) .
\end{aligned}
$$


Now applying (3.3) and (3.4) to (3.5), we have

$$
\begin{aligned}
\operatorname{per} B_{4, n}= & 16\left(b_{1} b_{n}+b_{1}^{2}+b_{n}^{2}\right) \operatorname{per} B_{4, n}(1,5, n) \\
& +\left(1-4\left(b_{1}+b_{n}\right)\right) \operatorname{per} B_{4, n}(5, n) \\
= & 16\left(b_{1}+b_{n}\right)^{2} \operatorname{per} B_{4, n}(1,5, n)-16 b_{1} b_{n} \operatorname{per} B_{4, n}(1,5, n) \\
& +\operatorname{per} B_{4, n}(5, n)-4\left(b_{1}+b_{n}\right) \operatorname{per} B_{4, n}(5, n) \\
= & -16 b_{1} b_{n} \operatorname{per} B_{4, n}(1,5, n)+\operatorname{per} B_{4, n}(5, n) \\
= & 4\left(b_{1}+b_{n}-4 b_{1} b_{6} n\right) \operatorname{per} B_{4, n}(1,5, n) .
\end{aligned}
$$

Thus if we increase $b_{1}$ and decrease $b_{n}$ with fixed $b_{1}+b_{n}$ in the above equation, then we can decrease $\operatorname{per} B_{4, n}$. This contradicts to the fact that $B_{4, n}$ is a minimizing matrix. Hence we must have $b_{1}=b_{n}$. Using $(3,2)$, we have that all $b_{i}$ and $x_{i}$ are the same, respectively. Thus $B_{4, n}$ with each $b_{i}=\frac{1}{n}$ and $x_{i}=\frac{n-4}{n}$ is a minimizing matrix on $\Omega\left(U_{4, n}\right)$, and it is the barycenter of $\Omega\left(U_{4, n}^{n}\right)$. Moreover, the minimum permanent is $\operatorname{per} b\left(\Omega\left(U_{4, n}\right)\right)=\operatorname{per} B_{4, n}(1 \mid 5)=\frac{4 !(n-1)(n-2)(n-3)(n-4)^{n-4}}{n^{n+3}}$ as required in $(3.1)$.

\section{Minimum permanents on $\Omega\left(V_{4, n}\right)$}

In this section, we consider the $(n+4)$-square $(0,1)$-matrix $V_{4, n}$ and determine the minimizing matrices and minimum permanents on the faces $\Omega\left(V_{4, n}\right)$ for $n \geq 6$. In [6], the authors gave the following conjecture:

Conjecture 4.1. ([6], Conjecture 2.1) $V_{m, n}$ is cohesive but not barycentric for $1<n<m+\sqrt{m}$, while for $n \geq m+\sqrt{m}, V_{m, n}$ is not cohesive and $b\left(U_{m, n}\right)$ is a minimizing matrix.

We show that this Conjecture 4.1 holds for the case $n \geq m+\sqrt{m}$, with $n=4$ and use contraction method to determine the minimum permanents on $\Omega\left(V_{4, n}\right)$.

Lemma 4.2. ([7]) For $n \geq 5$, the minimum permanent on $\Omega\left(V_{3, n}\right)$ is $\frac{6(n-1)(n-2)(n-3)^{n-3}}{n^{n+2}}$ and the unique minimizing matrix is the barycenter $b\left(U_{3}, n\right)$ of $\Omega\left(U_{3, n}\right)$. 
Lemma 4.3. For $n \geq 5$, the minimizing matrix on $\Omega\left(V_{4, n}\right)$ is the form

$$
A_{4, n}=\left[\begin{array}{cc}
a J_{4,4} & d J_{4, n} \\
d J_{n, 4} & c I_{n}
\end{array}\right]
$$

with $4 a+n d=1=4 d+c$.

Proof. Let $A_{4, n}$ be a minimizing matrix on $\Omega\left(V_{4, n}\right)$. Since the first 4 columns and first 4 rows of $V_{4, n}$ are the same, we can use the averaging method on those columns and rows of $A_{4, n}$, respectively. Thus we can write $A_{4, n}$ as follows:

$$
A_{4, n}=\left[\begin{array}{c|cccc}
a J_{4,4} & \overline{\mathbf{d}}_{1} & \overline{\mathbf{d}}_{2} & \ldots & \overline{\mathbf{d}}_{n} \\
\hline \overline{\mathbf{d}}_{1}^{t} & c_{1} & & & 0 \\
\overline{\mathbf{d}}_{2}^{t} & & c_{2} & & \\
\vdots & 0 & & \ddots & \\
\overline{\mathbf{d}}_{n}^{t} & & & & c_{n}
\end{array}\right],
$$

where $\overline{\mathbf{d}}_{i}\left(\overline{\mathbf{d}}_{i}^{t}\right)$ is a column (row) vector with $d_{i}$ as all its entries for $i=$ $1,2, \cdots, n$. Since the permanent value is invariant under the interchange of rows (and columns), we may assume that

$$
d_{i+1} \leq d_{i}\left(\text { i.e. } c_{i+1} \geq c_{i}\right)
$$

for $i=1, \cdots, n-1$ without loss of generality. Since $V_{4, n}$ is fully indecomposable, we can have that

$$
d_{i}>0
$$

for $i=1, \cdots, n$ by Lemma 1.1 .

Suppose $c_{1}=0$. Then the fourth row and column of $A_{4, n}$ have exactly 4 nonzero entries. Thus we can obtain a generalized contraction $G C\left(A_{4, n}\right)$ of $A_{4, n}$. Since the third row of $G C\left(A_{4, n}\right)$ has exactly 3 nonzero entries, we can obtain its generalized contraction $G C\left(G C\left(A_{4, n}\right)\right)$, which is contained in $\Omega\left(V_{3, n-1}\right)$. Using Lemmas 2.1 and 2.2 , we have

$$
\begin{aligned}
\operatorname{per}\left(A_{4, n}\right) & =\left(\frac{3}{4}\right)^{3} \operatorname{per}\left(G C\left(A_{4, n}\right)\right) \\
& \geq\left(\frac{3}{4}\right)^{3}\left\{\left(\frac{3}{4}\right)^{3} b\left(U_{3, n-1}\right)\right\} \\
& =\left(\frac{3}{4}\right)^{6} \cdot \frac{6(n-2)(n-3)(n-4)^{n-4}}{(n-1)^{n+1}} .
\end{aligned}
$$


$\operatorname{But} \operatorname{per}\left(A_{4, n}\right)$ is less than or equal to the permanent of the barycenter $b\left(U_{4, n}\right)$. That is,

$$
\begin{aligned}
& \operatorname{per}\left(A_{4, n}\right) \leq \operatorname{per}\left(b\left(U_{4, n}\right)\right) \\
& =4 \cdot \frac{1}{n}\left[(n-1) \cdot \frac{1}{n} \cdot \frac{3}{n} \cdot(n-2) \cdot \frac{1}{n} \cdot \frac{2}{n} \cdot(n-3) \cdot \frac{1}{n} \cdot \frac{1}{n}\right]\left(\frac{n-4}{n}\right)^{n-4} \\
& =\frac{4 !(n-1)(n-2)(n-3)(n-4)^{n-4}}{n^{n+3}}
\end{aligned}
$$

If we divide the last value in (4.6) by the last value in (4.5), then we can show that the result is less than 1 by a direct calculation using Mathematica Program for $n \geq 5$. Thus we have a contradiction from the inequalities in (4.5) and (4.6). Hence $c_{1}$ is not zero. Hence all $c_{i}>0$ by (4.3) for $i=1, \cdots, n$.

Suppose to the contrary that $b_{1}>b_{n}$. Let

$$
\begin{aligned}
& Q_{1}=\operatorname{per} A_{4, n}(5, n+4), \\
& Q_{2}=\operatorname{per} A_{4, n}(1,5, n+4), \\
& Q_{3}=\operatorname{per} A_{4, n}(1,2,5, n+4) .
\end{aligned}
$$

From per $A_{4, n}(1 \mid 5)=\operatorname{per} A_{4, n}=\operatorname{per} A_{4, n}(1 \mid n+4)$, we have

$$
4 d_{1}\left\{\left(3 d_{n}\right)^{2} Q_{3}+c_{n} Q_{2}\right\}=4 d_{n}\left\{\left(3 d_{1}\right)^{2} Q_{3}+c_{1} Q_{2}\right\}
$$

and hence

$$
4 \cdot 9 \cdot d_{1} d_{n}\left(d_{n}-d_{1}\right) Q_{3}=4\left(c_{1} d_{n}-d_{1} c_{n}\right) Q_{2} .
$$

Using $c_{i}=1-4 d_{i}$, we obtain

$$
9 b_{1} b_{n} Q_{3}=Q_{2} .
$$

On the other hand, from

$$
\operatorname{per} A_{4, n}(5 \mid 5)=\operatorname{per} A_{4, n}=\operatorname{per} A_{4, n}(n+4 \mid n+4),
$$

we have

$$
c_{1} Q_{1}+\left(4 d_{1}\right)^{2} Q_{2}=c_{n} Q_{1}+\left(4 d_{n}\right)^{2} Q_{2} .
$$

Using $c_{i}=1-4 d_{i}$, we obtain

$$
Q_{1}=4\left(d_{1}+d_{n}\right) Q_{2}
$$

Let us calculate per $A_{4, n}$ by expanding along the 5 th and $(4+n)$ th columns. Then we have

$$
\begin{aligned}
\operatorname{per} A_{4, n} & =\left(4 d_{1}\right)^{2} \operatorname{per} A_{4, n}(1,5)+c_{1} \operatorname{per} A_{4, n}(5 \mid 5) \\
& =\left(4 d_{1}\right)^{2}\left\{\left(3 d_{n}\right)^{2} Q_{3}+c_{n} Q_{2}\right\}+c_{1}\left\{\left(4 d_{1}\right)^{2} Q_{2}+c_{n} Q_{1}\right\} .
\end{aligned}
$$


Using $c_{i}=1-4 d_{i}$ and applying (4.7), (4.8) to this equation, we have

$$
\begin{aligned}
\operatorname{per} A_{4, n}= & 16\left(d_{1} d_{n}+d_{1}^{2}+d_{n}^{2}\right) \operatorname{per} A_{4, n}(1,5, n+4) \\
& +\left\{1-4\left(d_{1}+d_{n}\right)\right\} \operatorname{per} A_{4, n}(5, n+4) \\
= & 4\left(d_{1}+d_{n}-4 d_{1} d_{n}\right) Q_{2} .
\end{aligned}
$$

Thus by increasing $d_{1}$ and decreasing $d_{n}$, with fixed the sum $d_{1}+d_{6}$, we can decrease per $A_{4, n}$. This is impossible since $A_{4, n}$ is a minimizing matrix. So we must have $d_{1}=d_{n}$, and hence all $d_{i}=d_{1}$ and all $c_{i}=c_{1}$ for $i=1, \cdots, n$.

Therefore we have shown the required form (4.1) with $4 a+n d=1=$ $4 d+c$ from (4.2) as the minimizing matrix on $\Omega\left(V_{4, n}\right)$ with $c_{i}=c$ and $d_{i}=d$ for $i=1, \cdots, n$.

Remark 4.4. In [6], the fact that $c_{i}>0$ was assumed in the proof of the Theorem 2.1 in [6]. But in order to determine the minimum permanents on $\Omega\left(V_{4, n}\right)$ in the following Theorem 4.5, we need to show that each $c_{i}>0$. We proved this fact in the above Lemma 4.3.

Theorem 4.5. For $n \geq 7$, the minimum permanent on $\Omega\left(V_{4, n}\right)$ is $\frac{4 !(n-1)(n-2)(n-3)(n-4)^{n-4}}{n^{n+3}}$, which occurs at the barycenter on $\Omega\left(U_{4, n}\right)$.

Proof. Let $A_{4, n}=\left[\begin{array}{cc}a J_{4,4} & d J_{4, n} \\ d J_{n, 4} & c I_{n}\end{array}\right]$ be a minimizing matrix on $\Omega\left(V_{4, n}\right)$ with $4 a+n d=1=4 d+c$ from Lemma 4.3. Since $n d \leq 1$, we have $d \leq \frac{1}{n}$. From $4 d+c=1$, we have

$$
c=1-4 d \geq 1-4 \cdot \frac{1}{n}=\frac{n-4}{n} \geq(n-4) d .
$$

Now, suppose to the contrary that $a \neq 0$. Then we have

$$
\begin{aligned}
0= & \operatorname{per} A_{4, n}(1 \mid 1)-\operatorname{per} A_{4, n}(1 \mid n+4) \\
= & (3 d)^{2} \operatorname{per} A_{4, n}(1,2, n+4)+(c) \operatorname{per} A_{4, n}(1, n+4) \\
& -(4 d) \operatorname{per} A_{4, n}(1, n+4) \\
= & \left(9 d^{2}\right) \operatorname{per} A_{4, n}(1,2, n+4)+(c-4 d) \operatorname{per} A_{4, n}(1, n+4) .
\end{aligned}
$$

Case 1) $n \geq 8$. Since per $A_{4, n}(1,2, n+4)$ and $\operatorname{per} A_{4, n}(1, n+4)$ are positive, equation (4.10) implies that $(c-4 d)$ must be negative. Then $c<4 d$. But $c \geq(n-4) d$ from (4.9). Thus $(n-8) d<0$ and $n<8$, which is a contradiction. Thus $a$ must be zero for $n \geq 8$. 
Case 2) $n=7$. Consider

$$
\begin{aligned}
0= & \operatorname{per} A_{4,7}(1 \mid 1)-\operatorname{per} A_{4,7}(1 \mid 11) \\
= & (3 d)^{2} \operatorname{per} A_{4,7}(1,2,11)+(c) \operatorname{per} A_{4,7}(1,11) \\
& -(4 d) \operatorname{per} A_{4,7}(1,11)
\end{aligned}
$$

If $c \geq 4 d$, we have a contradiction that the right of (4.11) is positive. Hence

$$
3 d \leq c<4 d
$$

from (4.9). And hence

$$
\frac{1}{8}<d \leq \frac{1}{7}, 0<a<\frac{1}{32}, \frac{3}{8}<c<\frac{4}{7}
$$

from doubly stochastic property of $A_{4,7}$. From (4.11), we have

$$
\begin{aligned}
0= & 9 d^{2} \operatorname{per} A_{4,7}(1,2,11)-d \operatorname{per} A_{4,7}(1,11) \\
& +(c-3 d) \operatorname{per} A_{4,7}(1,11) \\
= & 9 d^{2} \operatorname{per} A_{4,7}(1,2,11)-d\left\{3 a \operatorname{per} A_{4,7}(1,2,11)\right. \\
& \left.+18 d^{2} \operatorname{per} A_{4,7}(1,2,10,11)\right\}+(c-3 d) \operatorname{per} A_{4,7}(1,11) \\
= & \left(d^{2}-3 a d\right) \operatorname{per} A_{4,7}(1,2,11)+\left\{8 d^{2} \operatorname{per} A_{4,7}(1,2,11)\right. \\
& \left.-18 d^{3} \operatorname{per} A_{4,7}(1,2,10,11)\right\}+(c-3 d) \operatorname{per} A_{4,7}(1,11) \\
= & d(d-3 a) \operatorname{per} A_{4,7}(1,2,11)+\left[8 d ^ { 2 } \left\{4 d^{2} \operatorname{per} A_{4,7}(1,2,3,10,11)\right.\right. \\
& \left.\left.+c \operatorname{per} A_{4,7}(1,2,10,11)\right\}-18 d^{3} \operatorname{per} A_{4,7}(1,2,10,11)\right] \\
& +(c-3 d) \operatorname{per} A_{4,7}(1,11) .
\end{aligned}
$$

Now consider

$$
\begin{gathered}
d(d-3 a)>d\left(\frac{1}{8}-3 \cdot \frac{1}{32}\right)>0 \\
8 d^{2} c-18 d^{3}=d^{2}(8 c-18 d)>d^{2}\left(8 \cdot \frac{3}{8}-18 \cdot \frac{1}{7}\right)=\frac{3}{7} d^{2}>0
\end{gathered}
$$

and

$$
c-3 d \geq 0
$$

from (4.12) and (4.13). These values show that the last equation of (4.14) is positive, which is impossible. Hence $a$ must be zero.

Thus the minimizing matrices on $\Omega\left(V_{4, n}\right)$ have the form $A_{4, n}$ in (4.1) with $a=0$ for $n \geq 7$. Therefore we have obtained that the minimum permanent on $\Omega\left(V_{4, n}\right)$ is $\frac{4 !(n-1)(n-2)(n-3)(n-4)^{n-4}}{n^{n+3}}$, which occurs at the barycenter $b\left(U_{4, n}\right)$ of $\Omega\left(U_{4, n}\right)$ by Theorem 3.1 for $n \geq 7$. 
Theorem 4.6. The minimum permanent on $\Omega\left(V_{4,6}\right)$ is

$$
\frac{4 ! 5 \cdot 4 \cdot 3 \cdot 2^{2}}{6^{9}} \approx 0.00057155921353452217650,
$$

which occurs at the barycenter $b\left(U_{4,6}\right)$ of $\Omega\left(U_{4,6}\right)$.

Proof. Let $A_{4,6}$ be a minimizing matrix on $\Omega\left(V_{4,6}\right)$. Then we have $A_{4,6}=$ $\left[\begin{array}{cc}a J_{4,4} & d J_{4,6} \\ d J_{6,4} & c I_{6}\end{array}\right]$ with $4 a+6 d=1=4 d+c$ from Lemma 4.3 .

Assume that $a \neq 0$.

Then we have per $A_{4,6}(1 \mid 1)=\operatorname{per} A_{4,6}(1 \mid 10)$ from Lemma 1.1.

Now, consider

$$
\begin{aligned}
\operatorname{per} A_{4,6}(1 \mid 1)= & 9 d^{2} \operatorname{per} A_{4,6}(1,2,10)+c \operatorname{per} A_{4,6}(1,10) \\
= & 9 d^{2}\left(2 a^{2} c^{5}+20 a d^{2} c^{4}+40 d^{4} c^{3}\right) \\
& +c\left(6 a^{3} c^{5}+90 a^{2} d^{2} c^{4}+360 a d^{4} c^{3}+360 d^{6} c^{2}\right),
\end{aligned}
$$

and

$$
\begin{aligned}
\operatorname{per} A_{4,6}(1 \mid 10) & =4 d \operatorname{per} A_{4,6}(1,10) \\
& =4 d\left(6 a^{3} c^{5}+90 a^{2} d^{2} c^{4}+360 a d^{4} c^{3}+360 d^{6} c^{2}\right) .
\end{aligned}
$$

If $c \geq 4 d$, then per $A_{4,6}(1 \mid 1)>\operatorname{per} A_{4,6}(1 \mid 10)$ from (4.15) and (4.16). This is a contraction. Thus $c<4 d$. Since $a \neq 0$, we have $6 d<1$, and hence $d<\frac{1}{6}$. From $4 d+c=1$, we have $1-4 d=c<4 d$. Hence $\frac{1}{8}<d<\frac{1}{6}$. By substituting $c=1-4 d, a=\frac{1-6 d}{4}$ to (4.15) and (4.16), we get two equal equations with one variable $d$. By using the Mathematica Program, we get

$$
\begin{aligned}
0= & \operatorname{per} A_{4,6}(1 \mid 1)-\operatorname{per} A_{4,6}(1 \mid 10) \\
= & 9 d^{2} \operatorname{per} A_{4,6}(1,2,10)+c \operatorname{per} A_{4,6}(1,10)-4 d \operatorname{per} A_{4,6}(1,10) \\
= & 9 d^{2}\left(2 a^{2} c^{5}+20 a d^{2} c^{4}+40 d^{4} c^{3}\right) \\
& +c\left(6 a^{3} c^{5}+90 a^{2} d^{2} c^{4}+360 a d^{4} c^{3}+360 d^{6} c^{2}\right) \\
& -4 d\left(6 a^{3} c^{5}+90 a^{2} d^{2} c^{4}+360 a d^{4} c^{3}+360 d^{6} c^{2}\right) \\
= & \frac{3}{32}-\frac{69}{16} d+\frac{753}{8} d^{2}-\frac{5049}{4} d^{3}+11355 d^{4}-70536 d^{5}+300576 d^{6} \\
& -841824 d^{7}+1396224 d^{8}-1036800 d^{9} \\
> & 0
\end{aligned}
$$

for $\frac{1}{8}<d<\frac{1}{6}$.

This contradiction shows that $a=0$. 
Thus the minimizing matrix on $\Omega\left(V_{4,6}\right)$ has the form $A_{4,6}$ with $a=0$. Therefore we have obtained that the minimum permanent on $\Omega\left(V_{4,6}\right)$ is $\frac{4 ! 5 \cdot 4 \cdot 3 \cdot 2^{2}}{69}$, which occurs at the barycenter $b\left(U_{4,6}\right)$ of $\Omega\left(U_{4,6}\right)$ by Theorem 3.1 for $n=6$. Using the Mathematica Program, we get the approximative value 0.00057155921353452217650 .

Hence we have determined minimizing matrices and minimum permanents on $\Omega\left(V_{4, n}\right)$ for $n \geq 6$. Also this results show that the Conjecture 4.1 is true for the case $V_{4, n}$ with $n \geq 6$.

\section{References}

[1] R. A. Brualdi, An interesting face of the polytope of doubly stochastic matrices, Linear and Multilinear Algebra, 17 (1985), 5-18.

[2] R. A. Brualdi and B. L. Shader, Minimum permanents on special faces of the polytope of doubly stochastic matrices, Linear Algebra Appl. 201 (1994), 103-111.

[3] G. P. Egorycev, The solution of the van der Waerden problem for permanents, Dokl. Akad. Nauk. SSSR 258 (1981), 1041-1044.

[4] D. I. Falikman, A proof of vander Waerden's conjecture on the permanent of a doubly stochastic matrix, Mat. Zametki 29 (1981), 31-38, Translated in Math. Notes 29 (1981), 475-479.

[5] T. H. Foregger, On the minimum value of the permanent of a nearly decomposable doubly stochastic matrix, Linear Algebra Appl. 32 (1980), 75-85.

[6] K. Pula, S. Z. Song and I. M. Wanless, Minimum permanents on two faces of the polytope of doubly stochastic matrices, Linear Algebra Appl. 434 (2011), 232-238.

[7] S. Z. Song, Minimum permanents on certain faces of matrices containing an identity submatrix, Linear Algebra Appl. 108 (1988), 263-280.

[8] S. Z. Song, Minimum permanents on a face of the polytope of doubly stochastic matrices, Linear Algebra Appl. 259 (1997), 169-182.

[9] S. Z. Song, Minimum permanents and minimizing matrices on certain doubly stochastic matrices, Linear Algebra Appl. 162-164 (1992), 786-790.

Seok-Zun Song

Department of Mathematics, Jeju National University,

Jeju 690-756, Republic of Korea.

E-mail: szsong@jejunu.ac.kr 